\title{
Analysis of AHP by BIBD
}

\author{
Iwaro Takahashi \\ Institule of Sucio- Ecousumic Mannung \\ finiversily of Tsukuba
}

\section{ABSTRACT}



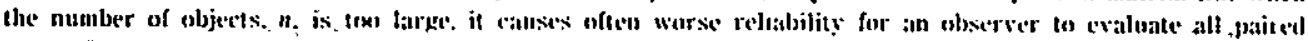

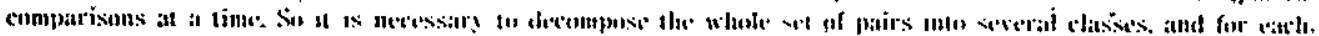

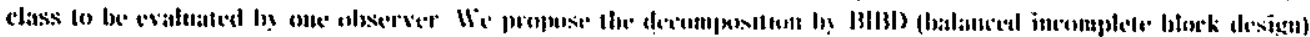

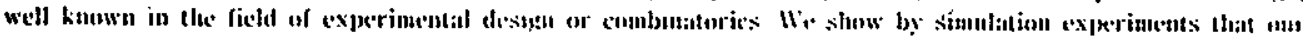

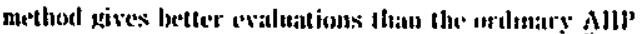

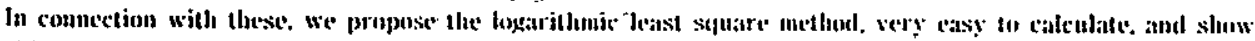
that this gives very gond approximation to the eigen vector anethod when $n$ is vather small. and alsat the former

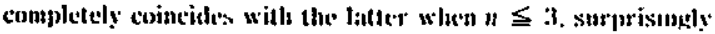

\section{$\$ 1$ Introduction}

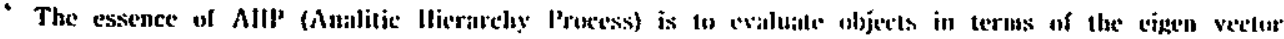

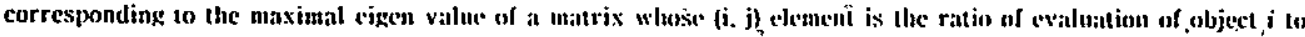

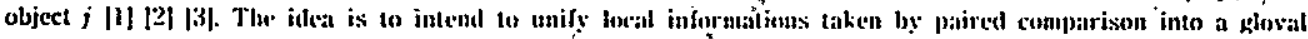
information.

But when tae number of objects is loo barke, it eatuses nften worse reliability far a observer to evaluate all paired comparisuns at a tine. In such case, it is meressary to tecompose tlu whole sel of paires of objects into several blocks, and for each block to be observed by one olsserser it is important how to decompose the set of

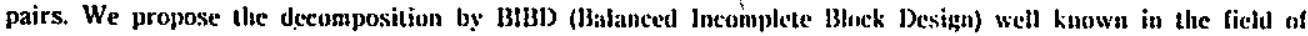
experinentaj design |4| [5]. And we show that this methol is to give betier evaluation under certain assumptions by simulation experiments (\$4). Further we propose the logarithnie least square melhod for our probletu, and show that this gives a good approximation (o) the eigen value method in NHP (\$3).

\section{$\S 2$ BiBD}

Let $E=|1,2, \ldots, v|$ be the set of objects $2=1.2, \ldots . n$ The immiber of pairs among $E$ is $C_{2}=v(v-1) / 2$. and if $v$ is large this becomes very large and an observer eannul compair all such pairs at at time. l.et the sel of objects which an observer can accomodate with sufficient reliability. be called an "illowable block"-, and let us denote the size of allowable block by $k(\leqq y)$.

Then we need to decompose the set of "C $C_{2}$ pairs into the classes of size ${ }_{\mathrm{k}} C_{4}$ and to allocale several observers to these classes. Each nbserver makes paired comparısans in- his class. We anify these results and can get the evaluation on $E$.

For example, there are $y=7$ applicants for a prize essay, and we try to judge their essays and to decidet ranking on them. f.et the allowable block sime le $k=3$, that is, one judge can rëad 3 essays and make paired

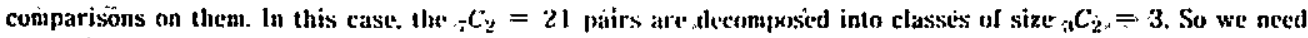
$21 / 3 \doteq 7$ judges. We unify the results of 7 judges snte the whole ranking on: 7 aspplicants.

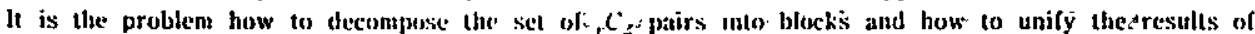
observations on blocks into the wlabe evaluation. We propxise the decompesition by BHBD and the unitication by the eigen value analysis used in AllP

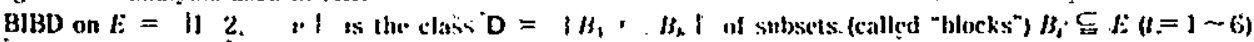
satisfying the followtngs:

, for any $t=1-b$

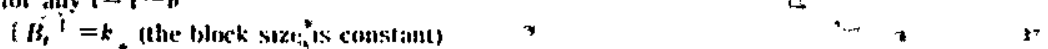

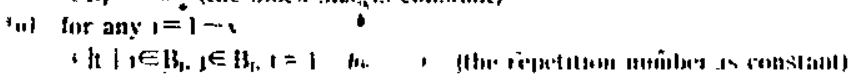


(where $|S|$ denotes the size of a set $S$.)

It is clear that (tii) implies (ii), so (i) and fiit) suffice for $D$ to be BIBD. Specifically a BIBD with $\lambda=1$ is called Steiner system and here we consider only Steiner system. which is denoted by (v. $k) \cdot \mathbf{D}$.

Example 1 The class of subsets of $E=|1,2, \ldots .7|$ shown in Table 1 is (7. 3)-D. The pairs in each block are shown in write, hand of the block. All such pairs construet the set of $; \mathrm{C}_{z}$ pairs. In other word. the set of pairs in $E$ is decomposed into pairs in blocks.

We can represent this situation in terms of graph theory in the following way: To construct ( $v . h$ ).D is equivalent to decompose the set of edges of a complete graph with $v$ points into complete graphs with $k$ points. ( $\rightarrow$ Fig. 1)

Table 1 (7. 3).D

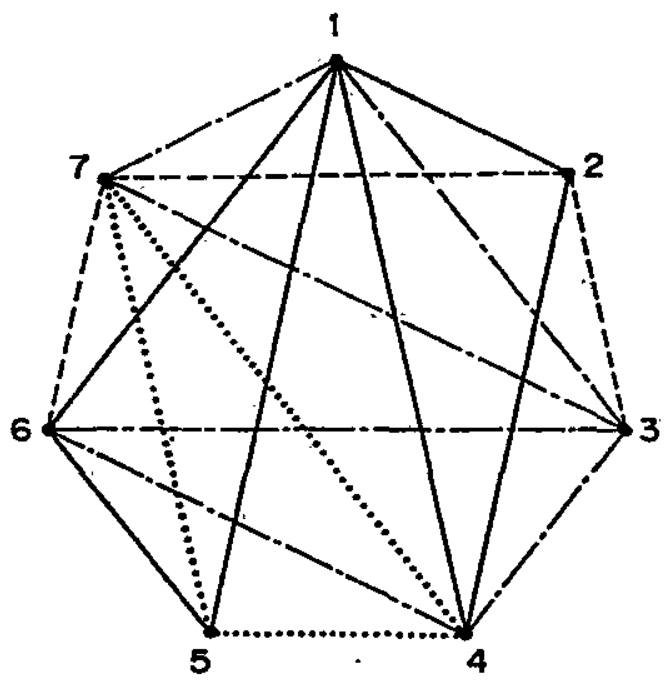

Fig. 1 BIBD decomposition

By the graph theoretic representation staded in Ex. 1. we can easily have the following relations.

$$
\begin{aligned}
& v r=\frac{b k}{C_{3}} \\
& b=\frac{v(v-1)}{{ }_{b} C_{3}}=\frac{v(k-1)}{H}
\end{aligned}
$$

If $(v, k) \cdot D$ exists then integers $y$ and $k$ satisfy. (2. 1). (2. 2) with integers $r$ and $b$, so for any integers $v$ and $k$ we do not necessarily have (v. k).D. For example (8, 3).D never exists. But (9. 3).D exists, so we can treal the case $v=8, k=3$ by taking oné of objects in (9.3):D as dumny.

The conditions of existence and construction methods of $(v, k) . D$ have been widely and decply rescarched in the field of experimental designs and combinatorial theories $[4][5]$.

In order to show why the decomposition by the (v. h).D is appropriale, we propose another rather natural decomposition shown in Table 2. Of course this $D=\left|B_{1}, \ldots, B_{7}\right|$ is not BIBD, where pair (1.,2) occurs 2 times in $B_{1}$ and $B_{7}$. while pairs (1. 4). (1. 5) do not occur anywhere.

The author believe that (v, k).D would give the best possible decompositions Inr our problems. In the end of this section we give another $(k, k) \cdot D$ in Table 3 .

Table 2

$$
\begin{aligned}
& B_{1}=|1.2 .3| \rightarrow \mid 2.13 .23 \\
& B_{2}=|2.3 .4| \rightarrow 23.24 .34 \\
& B_{10}=|3.4 .5| \rightarrow 3.35 .45 \\
& B_{1}=|1.5 .6| \rightarrow 45.46 .56 \\
& B_{6}=|6.6 .7| \rightarrow 56.57 .67 \\
& B_{6}=|6.7 .1| \rightarrow 67.61 .71 \\
& B_{5}=|7.1 .2| \rightarrow 71.7212
\end{aligned}
$$




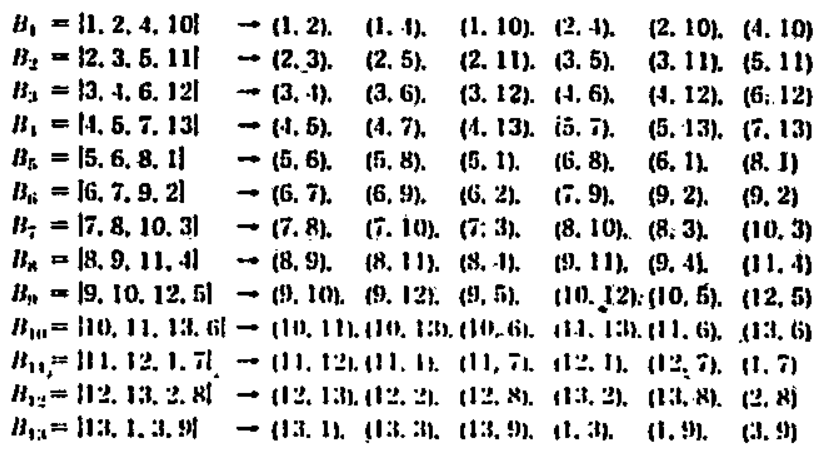

83 LLS. AlIP estimation

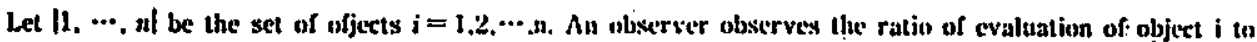
object $j$ and let us denote the observation by $x_{i j}$.

We assume the statistical model of $x_{i j}$ to be

$$
\begin{aligned}
& x_{i j}=a_{i j} \cdot e_{i j} . \quad a_{i j}=w_{i} / x_{j i} \quad(i<j, i, j=1-n) \\
& x_{j i}=b / x_{i j}
\end{aligned}
$$

Where $w_{i}(>0)$ is the real craluation of object $i$ and is an unknown parameter, and $c_{i j}(>0)$ is an idependent random variable representing the error of the observation. And we always recognize that any multiples of $[w, \cdots$ $\left.w_{n}\right]$ are equivalent to $\left[w_{1}, \cdots w_{m}\right]$ itself. Further we assume that

$$
E\left(n e_{i j}\right)=0, \quad V\left(n n e_{i j}\right)=\sigma^{2}(n)
$$

and $\sigma^{z}(n)$ is a monotone increasing function of $n$, the number of objects to be observed.

Whether these assumptions are reasonable or nol is a psychological or a physiological problem. but we can agree with these assumptions as a trial scheme.

The main purpose of AHP is to get estimales $w_{f}$ of $w_{i}(i=1-n)$ by calculating the cigen vector $w=\mid w_{1}, \cdots$. $\dot{w}_{n} \mid$ corresponding to the maximal eigen valne $\lambda$ of the $n \times n$ comparison matrix $X=\left|x_{j}\right|$.

Of course we have other extimation methods. The most natural one is "lognrithmic least square. (LLS)!. For simplicity let $\vec{x}_{i j}=\ln x_{i j}, \vec{w}_{i}=\ln w_{i}$ and $\bar{e}_{i j}=\ln e_{i j}$. Then we have

$$
\bar{x}_{j j}=\bar{w}_{i}-\bar{x}_{j}+\bar{e}_{i j} \quad(i<j, i, j=1 ; n)
$$

Appling the least square method to(3 - 3)we have last last square estimate $\vec{w}_{j}$ of $\vec{w}_{j}$, and taking inverse transform we have $w_{i}=e^{\#}(i=1-n)$. This is LLS estimation.

For example let $n=3$. Then we have

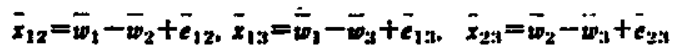

As the vector $w=f w_{1}, \cdots, w_{n} \mid$ muttiplied by an arbitral constant is equinalent to $w$ itself, We can assume $w_{1} w_{2} w_{3}=1$, so we have

$$
\bar{w}_{1}+\bar{w}_{2}+\bar{w}_{3}=0
$$

Appling least square method to $(3-4),(3-5$ )and taking inverse transtorm we have

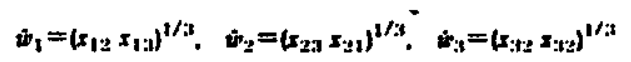


This estimation is very simple. but we have a surprising fact that $w_{0}(i=1-3)$ in $(3 \cdot 6)$ counctle with the components of the eigenvector corresponding to the maximal eigenvalue of the comparison natrix $X=\left|x_{n !}\right|$ That is. we have

Theorem 1 In the case $n \leq 3$. LLS estimates coincide with AlIP estinates.

Proof In case of $n=2$, we have easily

$$
\dot{w}_{1}=\sqrt{x_{12}} . \quad \dot{w}_{2}=\sqrt{x_{2: 1}}
$$

as the LLS estimates of $\mathrm{w}_{1}$, $w_{2}$ respectively. And by direct calaculation we have

$$
x \quad\left[\begin{array}{l}
w_{1} \\
w_{i}
\end{array}\right]=2\left[\begin{array}{l}
w_{1} \\
w_{1}
\end{array}\right] \quad x=\left[\begin{array}{ll}
1 & x_{12} \\
x_{11} & 1
\end{array}\right]
$$

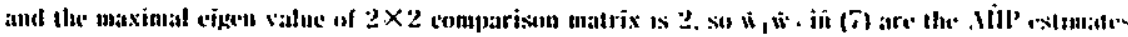

In case of $n=3$. from (6) we have

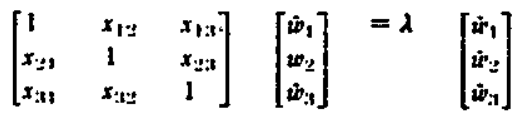

$$
\begin{aligned}
& \lambda=1+r+1 / r, r=* V_{x_{1}, 2} x_{2: 3} x_{: 31}
\end{aligned}
$$

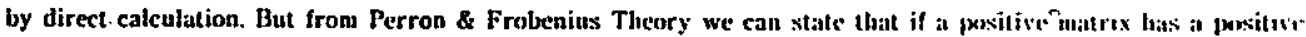


whose components are all positive). (See the proof of the theorem $f$ in $\mid$ ti $\mid$.

Like the case $n=3$ we have gereral LL.S estimal's

$$
\dot{w}_{i}=\left(\Pi_{i=1}^{n} x_{i j}\right)^{1 / n} \quad i=1, \cdots, n
$$

.which can be siated simply as $\bar{w}_{b}$ is the geometric mean of $i \cdot t h$ row of the comparison matrix $X$

But unfortunately they no longer coincide with AlfP estimates for $n>3$. Thus if $n>3$ then Theor $\mathrm{n}$ I dous

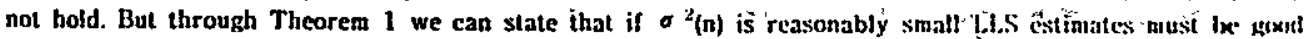
approximations to AHP estimates even $\mathrm{if} n$ is greater than 3. This also teaches us that our stitistital nursclel (a) 1 ) is valid for the AlIP analysis.

$\$ 4$ Decomposition Methods by BIBD

Now we propose our decomposition methods by BIBD, We are given the set of objects $l=11,2, \cdots, m$ to br evaluated. Let the allowable block size $k$ be far smaller than $v$ We decompose $E$ into blocks $B_{1}$. $\cdots$ ih $h_{h}$ which construct Steiner system (v. k).D.

Step 1 For each $B_{1}=\left|\beta_{1} . \beta_{2}, \cdots, \beta_{k}\right| \subseteq E$ an observer observes the objects and goîts obiscrvation $x$, by a

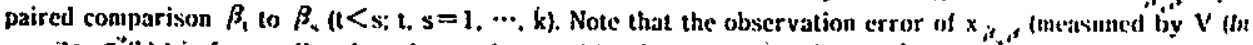
$\left.x_{\beta, \beta}\right)=\sigma^{*}(k)$ ) is far smaller than the one incurred by the observation in the whole sit $\mathrm{F}$

Step 2 l.et

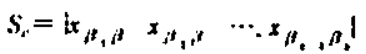

and let

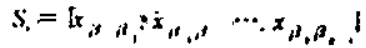

$$
\begin{aligned}
& =11 / x_{i f, a}, 1 / x_{, t, a}, \cdots, \mid / x_{B,}, 1 \\
& \text { fur } a=1 \quad 2, \cdots
\end{aligned}
$$

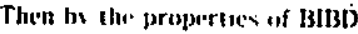

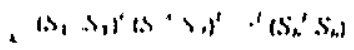





matrix $X=\left|x_{n}\right|$

Step 3 We apply the usual AlIP to the comparnsen matrix $x$. that is. we calculate the ciggen sectos $\dot{t}=\left[\dot{w}_{1}, \dot{u}_{2}, \ldots, \dot{w}_{r} \mid\right.$

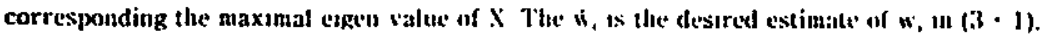

Example $3 \quad(v=7 \quad k=3$. BIBD decompositition)

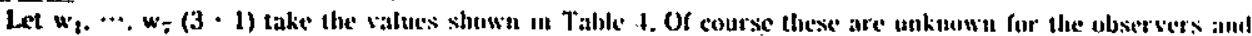
are to be estimated. Further $a_{n}=u_{,} / u_{r}\left(i_{.} j=1-\pi\right)$ are atso sluwn in Table 1.

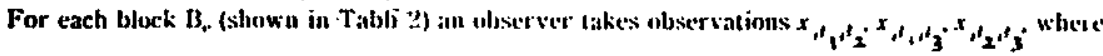

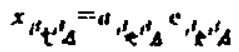

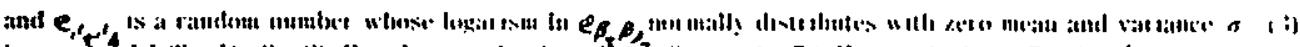

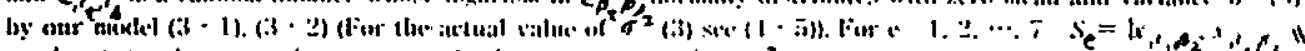

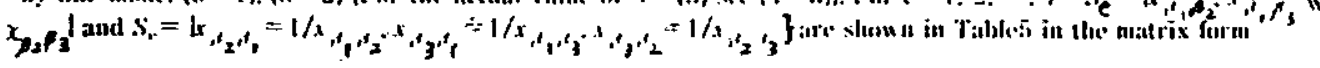

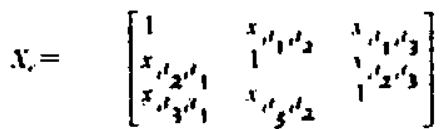

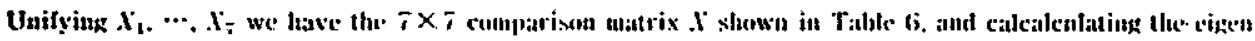

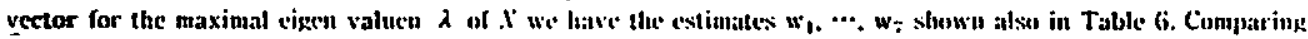

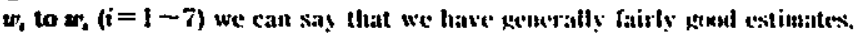

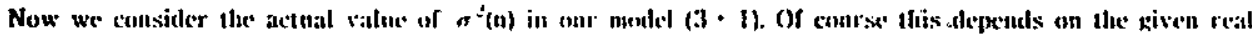
problen. I3ut we :ısum

$$
\sigma(3)=0.158^{*} . \sigma^{2}(4)=0.250 . \sigma^{2}(7)=0.5^{*} . \sigma^{\prime}(13)=0.980^{2}
$$

as trial values in our sinulationts. where $\sigma^{\circ} \cdot(n)$ is rouglily projxutinal $(1){ }_{n} C_{2}$.

Example $4 \quad(v=7$, direct ntethed $)$

Here we will describe the ustal Alt' netluxl asplised directly to Table 4. We multply $a_{1}$ by a random number $e_{i j}$ and have

$$
x_{a j}=a_{n} c_{n} \quad(i<j, i, j=1, \cdots .7)
$$

where Ine, normally distributes with zero mealt and variance $\sigma "(7)=0.5(-\infty, 5))$. and calculate $x_{n}=1 / x_{11}$ then we have the comparison matrix $X=\left|x_{1}\right|$ shown in Table 7 . Calculation the eigen vector for the maximal eigen value $\lambda$ we have eștinetes $\hat{\omega}_{i}(i=1, \cdots$. 7$)$ shown alson in Table 7. quite worse than the ones in Table 6 .

Next we try to investigate another case $v=13, k=4$ in lixamples '5, 6 along llue sane line as above. Example $5 \quad(v=13, k=4$. I3IBD decomposition)

We show $w_{i}(i=1-13)$ and $a_{1 j}=w_{i} / w,(i . l=1-13)$ in Table 8 . and(13, 1$)-\mathrm{D}$ and its comparisom abservations in Table 9, where the logarisn of the randum menbers bave' the variance $\sigma^{2}(4)=0.250^{2}$ (in $(4 \cdot 5)$ ) Fimally the unified comparison matrix $X$ and its maxinal eject value and the corresponding eigen vector are shown in Table 10 . These give us rather good estimates.

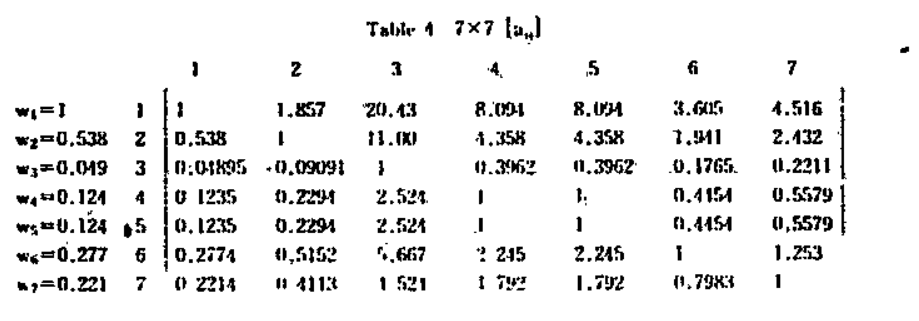




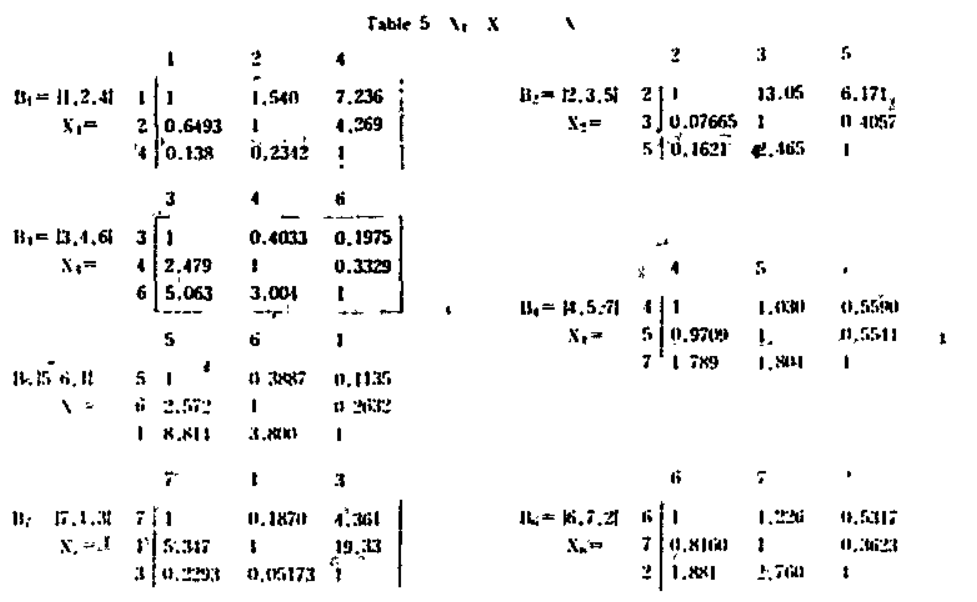

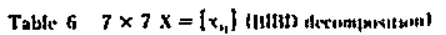

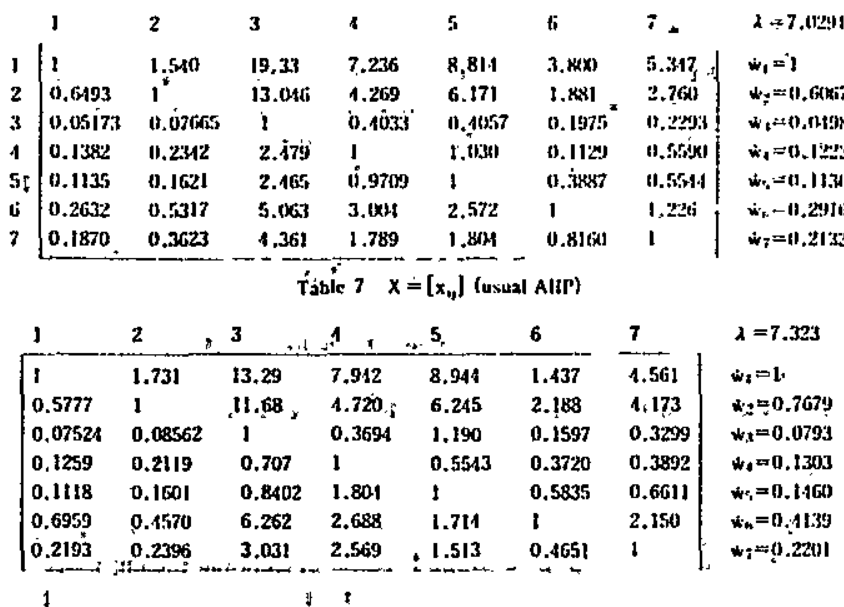

Example (j $(v=13$. direct method)

If an olserver observes , $C_{2}$ paired comparisons $x_{i j}(i<j ; i . j .=1-13$ ) at a time. then the logenithm of

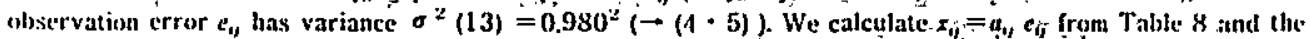

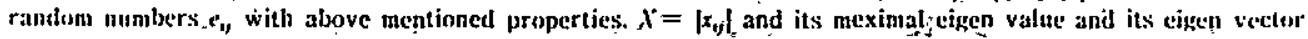
:He show

$\Lambda$ the end of this section we nóle that we can ușe. Li.S estimation $(-\$ 3)$ for our purpose:-

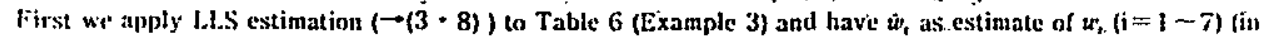
rable (1). These are slown in Table 12, where $w_{i}$ (in Table 6) are shown again for the conparison. (O) course $\dot{u}$, ;

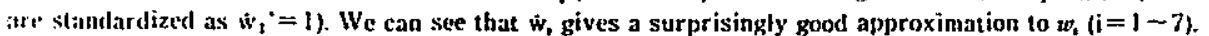

Sccoud appling L.I.S to Table 7 we have Table 13. This also gives farely good approximations. Next we have I thlle 1.1 from Table 10 and Table 15 from Tabic 11 by the same way as above.

Generally we can say that the L.I.S estimation gives a goxd approximation to the cigen vector estimation when the ilserervation error is small and the number of abjects is small.

The baber of the calculation af ale LLS is far easier than the eigen vector method. The forroes is easily deme. at a diest calculatur of pocket size, but the latter needs at least a personal computer so the adviantege of t.l.s

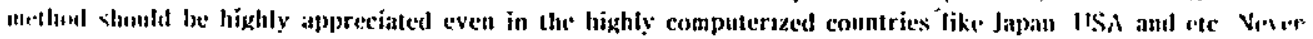

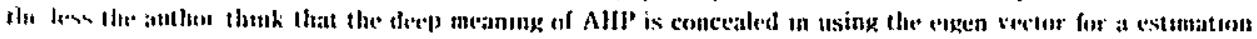






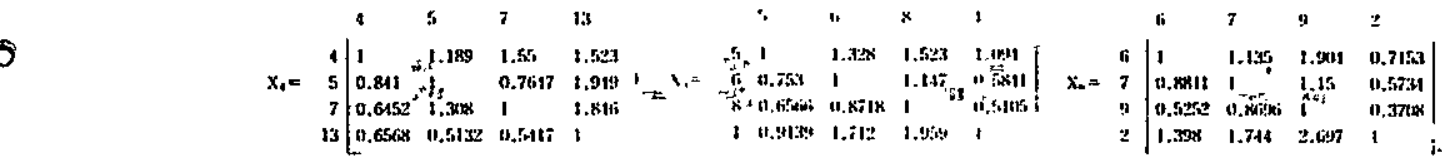

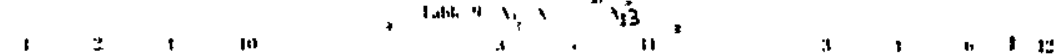



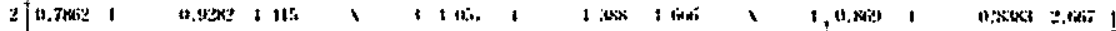

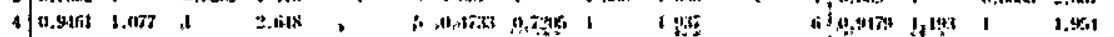

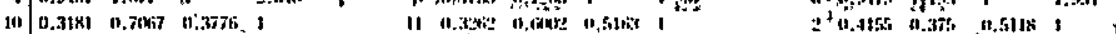

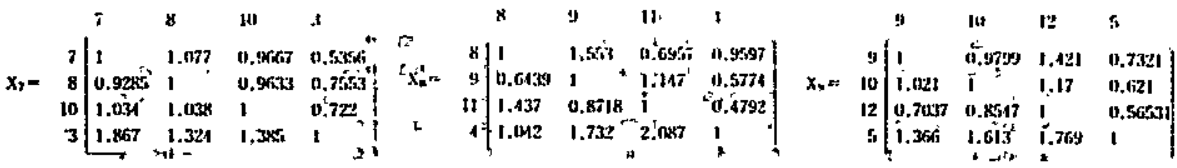

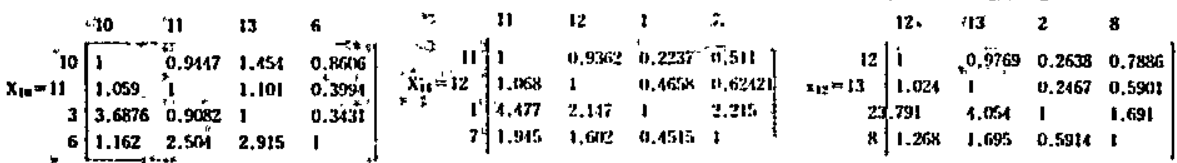

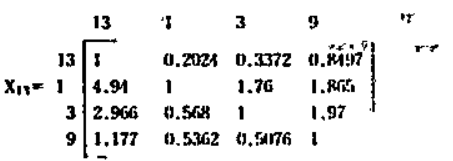

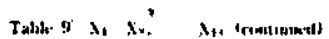

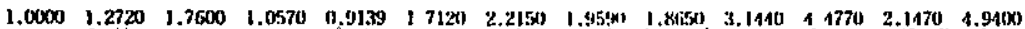

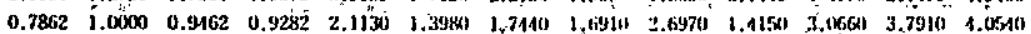

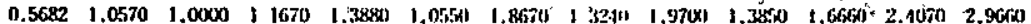

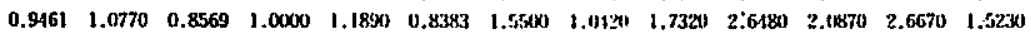

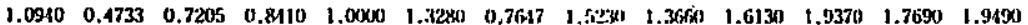
$\begin{array}{llllllllllllll}0.5841 & 0.7153 & 0.9479 & 1.1930 & 0.7530 & 1.0000 & 1.33501 & 1 & 14711 & 1.9041 & 1.1620 & 2.5040 & 1.9540 & 2.91501\end{array}$

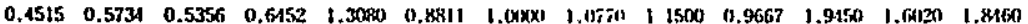
$\begin{array}{llllllllllllll}0.5105 & 0.5914 & 0.7553 & 0.9597 & 0.6566 & 0.8718 & 0.9285 & 1 & (6) 191 & 1.5630 & 0.9633 & 0.6957 & 1.2650 & 1.6950\end{array}$ $\begin{array}{lllllllllllllll}0.5362 & 0.3708 & 0.5076 & 0.5774 & 0.7321 & 0.5252 & 0.86696 & 0.5139 & 1 .(\times \times \times 0 & 0.9769 & 1 & 1470 & 1.1210 & 1 & 1751\end{array}$

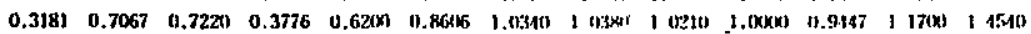

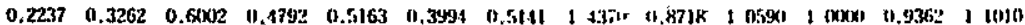

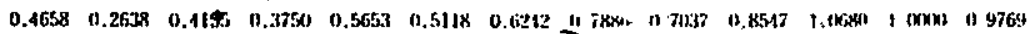

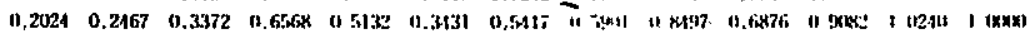

- arerncaluer 18:3131zo

- irstire






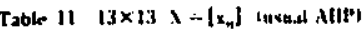

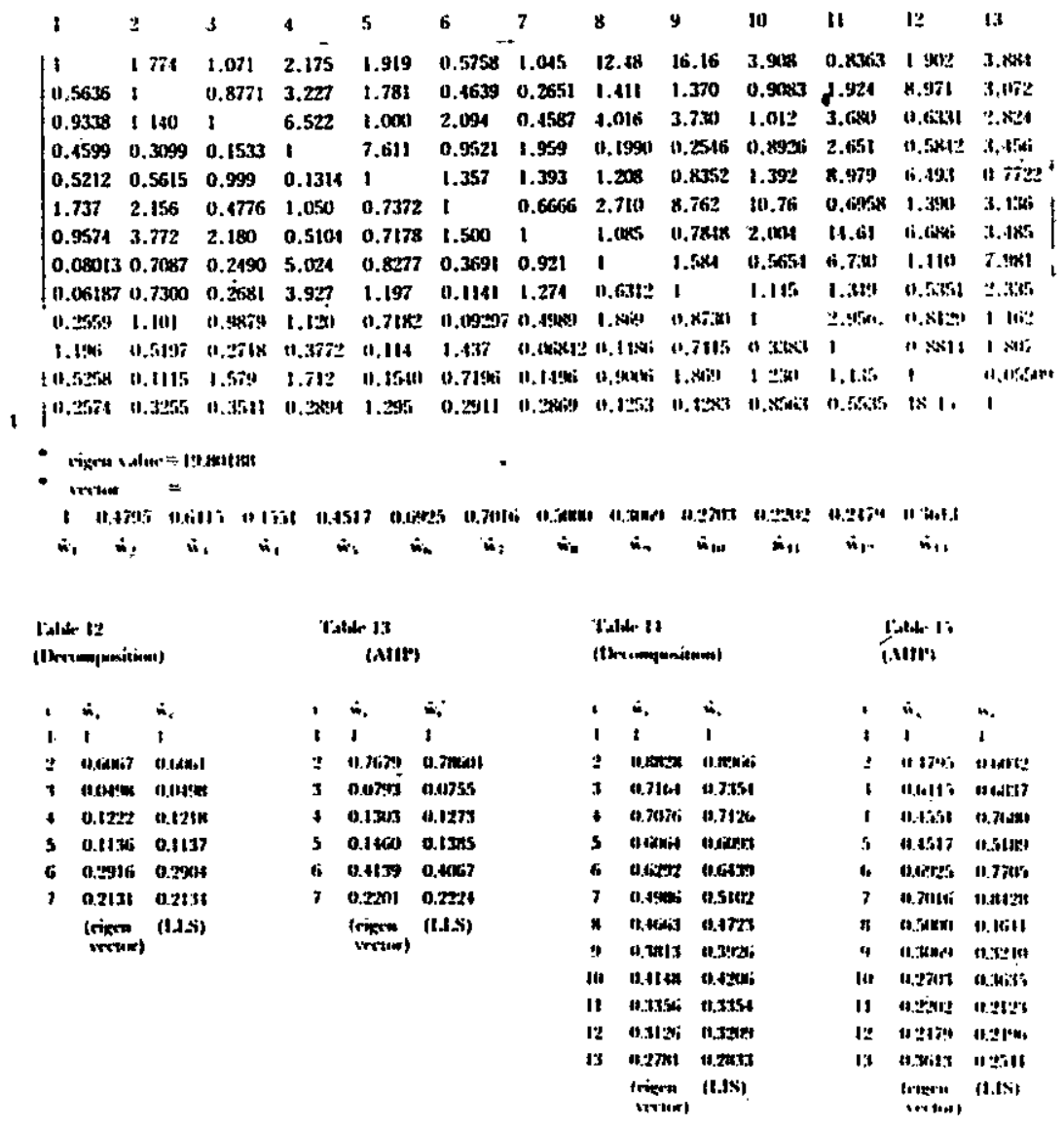

\section{Conclutions}

For the statistical model stated in $(3 \cdot 1)(3 \cdot 2)$ our BIBD methog gives better results than the usual AllP method. Further the L.LS method $(3-8)$ ) gives very gorsl approximation to the eigen value incthusl.

\section{References}

[1] Saaty. T.L.. A Scaling Method for Priorities in Hierarchical Structures, J. of Matlematical P'sychulogy. 15. pp. $234-281$ (1977)

[2] Saaty. T.L.. The Analytic llierarchy Process, McGraw-Hill (1980)

[3] Manabe. R. AHP(Analytic Hierarchy, Process) (in Japanese). Communi catings of the O.R Sucuty of Japan. 31. pp.45T-478 (1986)

[1] Hall. M: Combinatorial Theory. Blais dell (1967)

[5] Beth. T.. Jumgnickel. D, and Leng. II Design Theory. Cambridge Unu Press (1!)(i)

[6] Takaliaslu. I' AHIP applied to binars and Ternary Comparisons. J of O.R Society af Jajaut (Submitted\} 